\title{
Holographic approach of the spinodal instability to criticality
}

\section{Maximilian Attems}

Theoretical Physics Department, CERN, CH-1211 Genève 23, Switzerland

Instituto Galego de Física de Altas Enerxías (IGFAE), Universidade de Santiago de Compostela, 15782 Galicia, Spain

E-mail: maximilian.attems@cern.ch

ABSTRACT: A smoking gun signature for a first-order phase transition with negative speed of sound squared $c_{s}^{2}$ is the occurrence of a spinodal instability. In the gauge/gravity duality it corresponds to a Gregory-Laflamme type instability, which can be numerically simulated as the evolution of unstable planar black branes. Making use of holography its dynamics is studied far from and near a critical point with the following results. Near a critical point the interface between cold and hot stable phases, given by its width and surface tension, is found to feature a wider phase separation and a smaller surface tension. Far away from a critical point the formation time of the spinodal instability is reduced. Across softer and harder phase transitions, it is demonstrated that mergers of equilibrated peaks and unstable plateaux lead to the preferred final single phase separated solution. Finally, a new atypical setup with dissipation of a peak into a plateau is discovered. In order to distinguish the inhomogeneous states I propose a new criterium based on the maximum of the transverse pressure at the interface which encodes phase-mixed peaks versus fully phase separated plateaux.

KEYWORDS: Holography and quark-gluon plasmas, Gauge-gravity correspondence, Black Holes in String Theory

ARXIV EPRINT: 2012.15687 


\section{Contents}

1 Introduction 1

2 Setup 2

3 Criterium for inhomogeneous states $\quad 6$

$\begin{array}{ll}3.1 & \text { Definition: peaks versus plateaux and gorges versus valleys }\end{array}$

3.2 Final stage 8

$\begin{array}{llr}3.3 & \text { Reshaping stage } & 9\end{array}$

$\begin{array}{ll}3.4 \text { Merger stage } & 10\end{array}$

4 Characteristics of the interface $\quad 11$

$\begin{array}{lll}4.1 \text { Shape } & 12\end{array}$

$\begin{array}{lll}4.2 & \text { Surface tension } & 13\end{array}$

5 Evolution of the spinodal instability $\quad \mathbf{1 3}$

$\begin{array}{ll}5.1 \text { Comparison of the formation time } & 13\end{array}$

$\begin{array}{lll}5.2 & \text { Peak dissipation into plateaux } & 15\end{array}$

$\begin{array}{lll}6 & \text { Discussion } & 17\end{array}$

\section{Introduction}

Analytical holographic studies pioneered the modeling of heavy-ion collisions with the emergence of collectivity [1-4]. Fast hydrodynamization, implying the early applicability of hydrodynamics to a relaxing fluid, got first established numerically in dynamical out-ofequilibrium holographic shock wave collisions [5-9] and further corroborated in the presence of bulk viscosity [10-18] to model the almost perfect fluid. As the Relativistic Heavy Ion Collider is accumulating plenty of experimental data in the ongoing beam energy scan [19] and the upcoming experimental Facility for Antiproton and Ion Research [20,21] will search the quantum chromodynamics phase diagram for the presumed critical point, theoretical studies on criticality garner new attention [22-38]. In the cool down of the formed quarkgluon plasma in hadronic collisions there is presumably a wide temperature range with large baryon chemical potential to hit the first-order phase transition line, whose endpoint is the critical point. A prime signal for such a first-order type phase transition is the spinodal instability. The gauge/gravity duality [39] opens up the possibility to study the dynamics of such a phase transition, as the holographic dual [40] of the spinodal instability is the Gregory-Laflamme instability [41-46], which is amenable to general relativity calculations.

In [47] we discovered a metastable inhomogeneous unstable solution and subsequently [48] found the phase separation of the spinodal instability. The spinodal instability 
develops in four stages: 1) exponential growth of the instability; 2) the reshaping; 3) the merger; 4) the preferred final solution [49, 50]. During the evolution of the spinodal instability one encounters different types of inhomogeneous states. With a field redefinition all stages of a strong spinodal instability and the hydrodynamization of shockwave collisions near a critical point were demonstrated to be described by hydrodynamics $[49,51]$. A recent analysis discusses the finite size effects [52] of the periodical longitudinal direction on the stability or instability of the plasma. A quite different setup involving plasma balls studies effects of the confined phase [53], but there is no spinodal region.

The purpose of this paper is to vary criticality in order to see the dynamical effects of different first-order phase transitions. For this endeavour a Gregory-Laflamme type instability is evolved on the gravity side to induce the spinodal instability on the gauge theory side. Strictly speaking, below the critical temperature the Gregory-Laflamme instability has only unstable regions. In the holographic construction a Gregory-Laflamme type instability is induced by the non-trivial potential of a weakly-coupled scalar field and is unstable only in a certain temperature range. To simplify the construction I consider a setup with no conserved charges, hence the critical point or the phase transition will lie on the temperature axes. Another distinction to quantum-chromodynamics besides the class of the phase transitions considered is the deconfined nature of the cold stable phase. The strength of the holographic approach is to be able to extract and simulate universal features of a two phase system in the limit of strong coupling. The focus of this paper is on the dynamical real-time features of the spinodal instability while approaching a critical point. Section 2 discusses the dual setup, in particular how to simulate on the gravity side the Gregory-Laflamme type instability: one evolves the Einstein equations in the bulk and reads off the boundary data describing the spinodal instability on the gauge theory side. If the first order phase transition occurs far from (near) the critical point one speaks of a strong (soft) first order phase transition. Section 3 introduces a new criterium which is used to distinguish the inhomogeneous states - plateaux, peaks, valleys and gorges - in the relevant stages of the spinodal instability (reshaping, merger and final). This allows us in section 4 to characterize for different criticality the shape and surface tension of the interface seen in the preferred and settled final states of the spinodal instability. In section 5 several dynamical real-time setups of the spinodal instability with varying criticality are demonstrated, including a newly revealed dissipative process of a peak into a plateaux. Finally, I discuss the implications of the varying criticality for the spinodal instability in section 6 .

\section{Setup}

The non-conformal bottom-up model [14,47] employed here, is described by Einstein Hilbert action coupled to a scalar with a non trivial potential $V(\phi)$

$$
S=\frac{2}{\kappa_{5}^{2}} \int d^{5} x \sqrt{-g}\left[\frac{1}{4} \mathcal{R}-\frac{1}{2}(\nabla \phi)^{2}-V(\phi)\right],
$$

with $\kappa_{5}$ the five-dimensional Newton constant. The chosen potential $V(\phi)$ is conformal in the ultra-violet, where the spacetime is Anti-de-Sitter (AdS), and has a minimum corre- 
sponding to an infrared fixed point in the gauge theory. The potential is derived from a superpotential [54]:

$$
L W(\phi)=-\frac{3}{2}-\frac{\phi^{2}}{2}-\frac{\phi^{4}}{4 \phi_{M}^{2}},
$$

where $L$ is the radius of the AdS solution in the ultra-violet. The first two terms in (2.2) are fixed by AdS and the dimension of the "dual" scalar operator. The third term is responsible for the non-conformality and the appearance of a critical point. The potential has a single parameter $\phi_{\mathrm{M}}$, whose value defines the transition type. For a subcritical value of $\phi_{\mathrm{M}}$ the transition is a first order phase transition, which then turns to a critical point, and finally for a supercritical value to a cross-over phase transition. This yields the following scalar potential

$$
L^{2} V(\phi)=-3-\frac{3}{2} \phi^{2}-\frac{1}{3} \phi^{4}+\left(\frac{1}{2 \phi_{\mathrm{M}}^{4}}-\frac{1}{3 \phi_{\mathrm{M}}^{2}}\right) \phi^{6}-\frac{1}{12 \phi_{\mathrm{M}}^{4}} \phi^{8},
$$

where the term $-\frac{1}{3 \phi_{\mathrm{M}}^{2}} \phi^{6}$ is responsible for critical behaviour.

In what follows, the numerical procedure outlined in $[18,55,56]$ is used and I will set the dimensionless parameter of the potential to the subcritical values ${ }^{1}$

$$
\phi_{\mathrm{M}}=\{2.25,2.3,2.35,2.4,2.45\} \text {. }
$$

Each theory set by a different value of (2.4) has a first order phase transition. The strongest phase transition (farthest from a critical point) in this setup occurs for $\phi_{\mathrm{M}}=2.25$, while the softest (nearest to a critical point), has $\phi_{\mathrm{M}}=2.45$ as one approaches the theory with critical point $\phi_{\mathrm{M}}^{*} \approx 2.521 .^{2}$ All the considered states are deconfined similar to the characteristic of the quark-gluon plasma. Note that the parameter $\phi_{\mathrm{M}}$ always appears as a square in the potential and therefore also in the equations of motion. Hence a small change in $\phi_{\mathrm{M}} \leq \phi_{\mathrm{M}}^{*}$ results in quite different theories and correspondingly in different equations of state.

In the trace of the stress tensor

$$
\left\langle T_{\mu}^{\mu}\right\rangle=-\Lambda\langle\mathcal{O}\rangle
$$

one recognizes the scale $\Lambda$, which sets the magnitude of the non-normalizable mode of the scalar field and is the source of the conformal invariance breaking. The rescaled stress tensor given by

$$
\left(\mathcal{E}, P_{L}, P_{T}, \mathcal{V}\right)=\frac{\kappa_{5}^{2}}{2 L^{3}}\left(-T_{t}^{t}, T_{z}^{z}, T_{x_{\perp}}^{x_{\perp}}, \mathcal{O}\right)
$$

omitting the expectation value signs, introduces the local energy density $\mathcal{E}$, the longitudinal pressure $P_{L}$, the transverse pressure $P_{T}$ and the expectation value of the scalar operator $\mathcal{V}$.

\footnotetext{
${ }^{1}$ Supercritical values of $\phi_{\mathrm{M}}$ lead to a cross-over phase transition with no spinodal instability, see [18] for the relaxation channels and out-of-equilibrium properties.

${ }^{2}$ It is numerically not practicable to get much closer to the critical point as due to the small momenta range of unstable modes the spinodal instability then needs exponentially longer to kick in.
} 

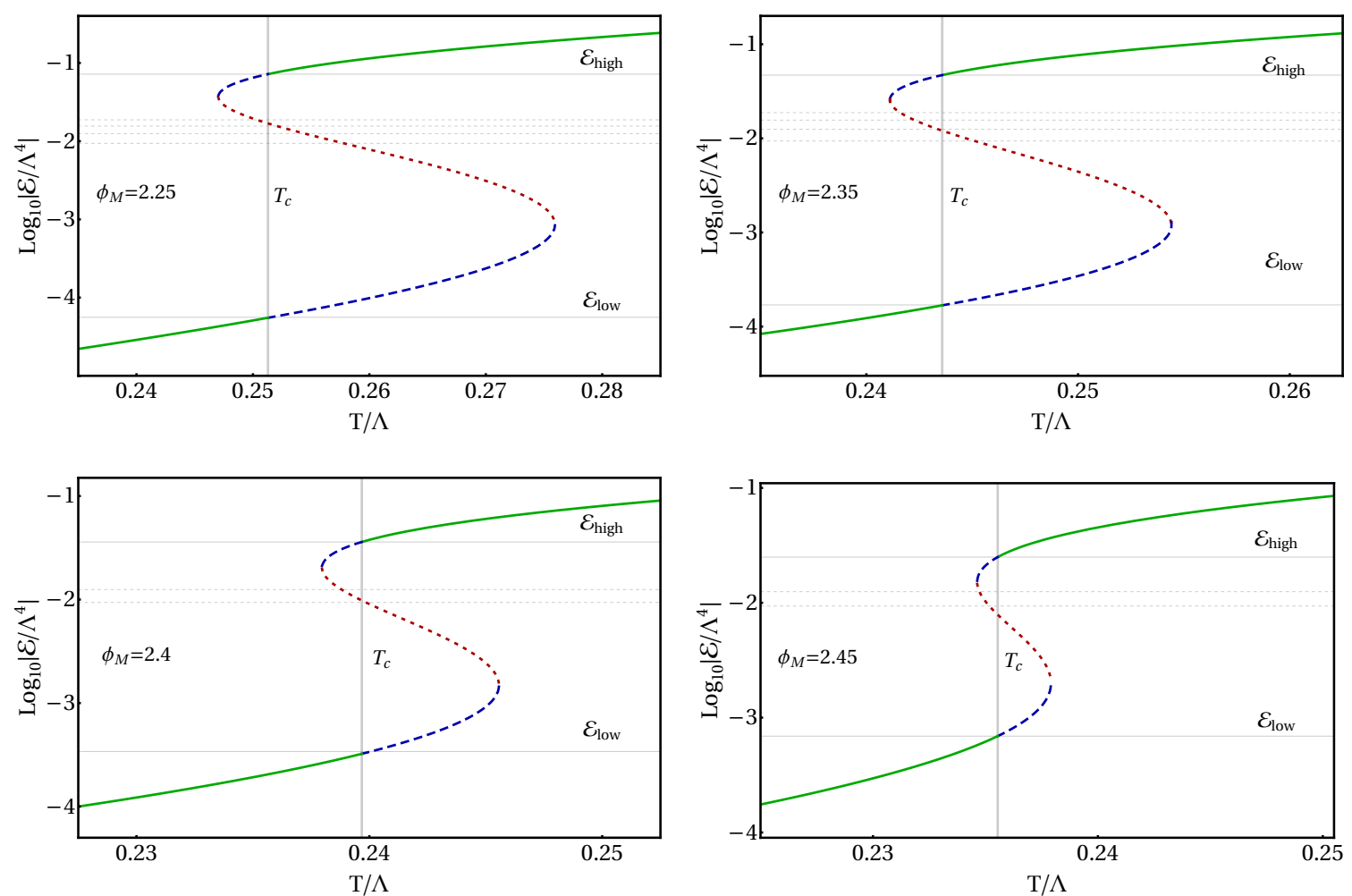

Figure 1. Energy density versus temperature for the theories with different first order phase transitions with varying criticality due to different parameter $\phi_{\mathrm{M}}=\{2.25,2.35,2.4,2.45\}$. The full green line represents the energy densities in the cold and hot stable phases, the dashed blue in the meta stable regions and the dotted red in the unstable region. The vertical full gray line marks the temperature of the phase transition $T_{c}$. The horizontal full grey lines mark the stable energy densities $\mathcal{E}_{\text {low }}$ and $\mathcal{E}_{\text {high }}$ at the transition temperature. The horizontal dashed grey lines are the four initial energy densities $\left\{\mathcal{E}_{1}, \mathcal{E}_{2}, \mathcal{E}_{3}, \mathcal{E}_{4}\right\}$ or just the two initial respective $\left\{\mathcal{E}_{1}, \mathcal{E}_{2}\right\}$ of the simulations.

Here $z$ is the dynamical and longitudinal direction, while $x_{\perp}$ are the transverse infinite homogeneous directions.

For the theories with values of $\phi_{\mathrm{M}}$ given in (2.4), I compute the relevant thermodynamical quantities. Figure 1 shows the energy density versus temperature with the additional indications of the local energy density of the stable high phase $\mathcal{E}_{\text {high }}$, the stable low energy phase $\mathcal{E}_{\text {low }}$, the transition pressure $P_{c}$ and the transition temperature $T_{c}$ (the thermodynamics of the theory with $\phi_{\mathrm{M}}=2.3$ has already been calculated [47, 49], so it is omitted in figure 1). As seen in figure 1 the thermodynamics depend crucially on the parameter $\phi_{\mathrm{M}}$ and each choice illustrates a different first-order phase transition. Going from $\phi_{\mathrm{M}}=2.25$ to $\phi_{\mathrm{M}}=2.45$, one notices the first order phase transition to become smoother and less pronounced. This results in the shrinking of the unstable region in figure 1, plotted in dashed red in the equation of state, both in the temperature and the local energy density range.

In table 1 are listed the respective $\mathcal{E}_{\text {high }}, \mathcal{E}_{\text {low }}, P_{c}$ and $T_{c}$ for each theory with varying first-order phase transition. In the section 3 , I will use the pressure of the transition $P_{c}$ to determine the difference between the inhomogeneous states. At the transition temperature 

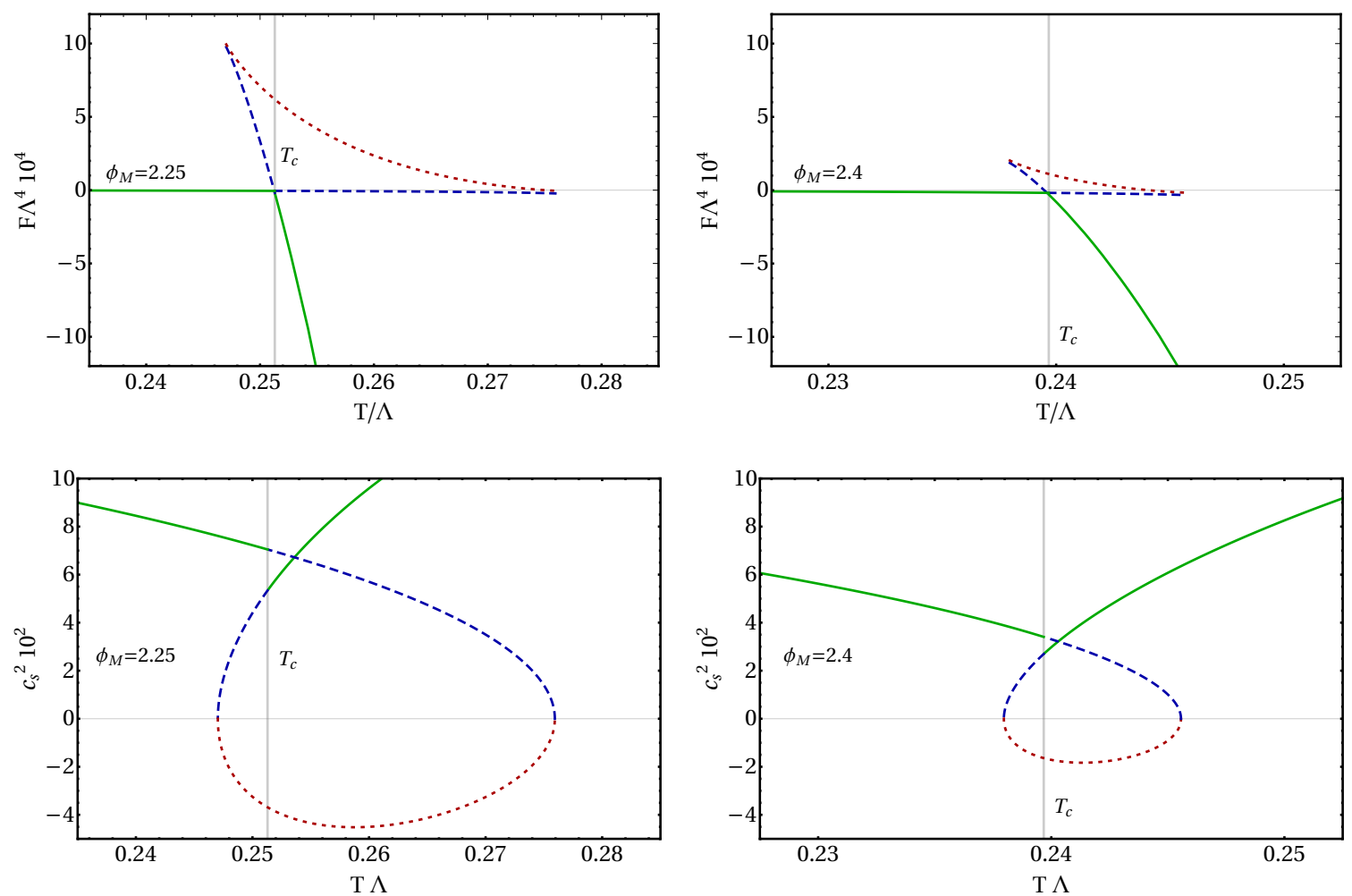

Figure 2. The free energy $F$ and the speed of sound squared $c_{s}^{2}$ over temperature for the theories with different first order phase transitions far from the critical point and closer to the critical point with varying criticality due to different $\phi_{\mathrm{M}}=\{2.25,2.4\}$. Each phase is denoted by the same color scheme as in figure 1.

\begin{tabular}{|l|l|l|l|l|l|}
\hline $\boldsymbol{\phi}_{\mathrm{M}}$ & 2.25 & 2.3 & 2.35 & 2.4 & 2.45 \\
\hline $\mathcal{E}_{\text {high }} / \boldsymbol{\Lambda}^{\mathbf{4}}$ & $7.2 \times 10^{-2}$ & $5.9 \times 10^{-2}$ & $4.7 \times 10^{-2}$ & $3.5 \times 10^{-2}$ & $2.5 \times 10^{-2}$ \\
$\mathcal{E}_{\text {low }} / \boldsymbol{\Lambda}^{4}$ & $5.6 \times 10^{-5}$ & $9.4 \times 10^{-5}$ & $1.7 \times 10^{-4}$ & $3.5 \times 10^{-4}$ & $6.9 \times 10^{-4}$ \\
$\boldsymbol{P}_{\boldsymbol{c}} / \boldsymbol{\Lambda}^{\mathbf{4}}$ & $0.3 \times 10^{-7}$ & $7.5 \times 10^{-6}$ & $0.7 \times 10^{-6}$ & $1.1 \times 10^{-6}$ & $2.9 \times 10^{-5}$ \\
$\boldsymbol{T}_{\boldsymbol{c}} / \boldsymbol{\Lambda}$ & 0.251 & 0.247 & 0.244 & 0.240 & 0.236 \\
\hline
\end{tabular}

Table 1. Local energy densities for the stable cold and hot phases of each theory and the corresponding transition pressures and temperatures.

$T_{c}$ homogeneous stable states have the same equilibrium pressure $P_{c}$. There are several ways to compute the transition temperature $T_{c}$. One way is via the crossing of the free energy $F$ as illustrated in the top plots of figure 2. For the energy densities in the unstable region between $\mathcal{E}_{\text {low }}$ and $\mathcal{E}_{\text {high }}$ - the spinodal region, the speed of sound squared is negative as plotted in the lower plots of figure 2 and the states are affected by a long-wave length instability. Note the stark difference between the two stable local energy densities from more than three to less than two orders of magnitude. Due to the more than three orders of magnitude difference between the stable cold and hot phases and due to the large spikes in gradients during the non-linear regime of the spinodal instability, the numerical real-time 
treatment of this system is extremely challenging. Between $\phi_{\mathrm{M}}=2.25$ and $\phi_{\mathrm{M}}=2.45$ the value for the hot stable phase $\mathcal{E}_{\text {high }}$ more than halves and the value for the cold stable phase $\mathcal{E}_{\text {low }}$ is approximately multiplied by twelve. The change in $\mathcal{E}_{\text {high }}$ is significant as the system with $\phi_{\mathrm{M}}=2.25$ compared to $\phi_{\mathrm{M}}=2.45$ needs more than twice as much total integrated energy density for a fully phase separated setup. Similarly, the change of magnitude in $\mathcal{E}_{\text {low }}$ is significant as it sets the behaviour of the phase domain wall. Moreover, it eases or hardens the numerical treatment. Obviously, there is a trade off between the needed numerical resolution for the stronger instability and the much longer evolution of the softer instability.

The setup uses homogeneous planar black brane solutions periodic in the $z$-direction with a finite box extent $L_{z}$ and different initial local energy densities: $\mathcal{E}(t=0)=\left\{\mathcal{E}_{1}, \mathcal{E}_{2}, \mathcal{E}_{3}, \mathcal{E}_{4}\right\}$. For the softer theories with $\phi_{M}=\{2.4,2.45\}$ and a smaller $\mathcal{E}_{\text {high }}$, it is sufficient to simulate setups with $\mathcal{E}(t=0)=\left\{\mathcal{E}_{1}, \mathcal{E}_{2}\right\}$. Moreover, for the theory with the parameter $\phi_{M}=2.45$ the bigger energy densities $\mathcal{E}_{3}$ and $\mathcal{E}_{4}$ are no longer in the unstable phase:

$$
\left\{\mathcal{E}_{1}, \mathcal{E}_{2}, \mathcal{E}_{3}, \mathcal{E}_{4}\right\}=\{0.9,1.2,1.6,1.9\} \times 10^{-2} \Lambda^{4} .
$$

In order to trigger the spinodal instability, one only has to slightly perturb these homogeneous solutions at the start of an evolution or wait for the numerical white noise to kick-in. Here I use a small sinusoidal perturbation with the amplitude of $\Delta=10^{-4}$ which, thanks to non-linear coupling, populates all unstable modes. This is much faster than numerical noise which needs to build up the unstable modes from amplitudes below $10^{-12}$. All the boundary data of the simulations is published for further analysis or comparisons as open data on a Zenodo repository [57]. As all the chosen initial local energy densities are in the dynamical unstable region between $\mathcal{E}_{\text {low }}$ and $\mathcal{E}_{\text {high }}$ of each theory, the plasma will be subject to the spinodal instability, whose dynamical properties one is interested in.

\section{Criterium for inhomogeneous states}

The spinodal instability cools down regions in the unstable energy density, so that they reach the cold stable phase and hence pushes out the energy density to other regions, which in turn accumulates and may reach the hot stable phase. This results in various inhomogeneous states, which are to be further classified. In what follows I will define the two distinct maxima and minima that form due to the spinodal instability: a peak and a plateau; a gorge and a valley.

\subsection{Definition: peaks versus plateaux and gorges versus valleys}

While the maxima domains, peaks versus plateaux, can be respectively defined by their total energy density content and the typical order of magnitude of difference between them, there is a more distinct and stringent criterium that applies when the maximum of the transverse pressure $P_{T}$ is near or overshoots the critical equilibrium pressure:

$$
\max \left(P_{T}\right) \gtrsim P_{c} \text {. }
$$

I will now discuss how this criterium can be used to define plateaux and valleys. 
Criterium for plateaux: if at the location of the maximum of the local energy density the transverse pressure is near or overshoots the critical equilibrium pressure eq. (3.1), the inhomogeneous state is a plateau.

The criterium originates from the observation that the transverse pressure at midpoint of smaller inhomogeneous states even in out-of-equilibrium stages never reaches the transition pressure $P_{c}$. On the other hand for the creation of plateaux one notices an overshoot, which is followed by an equilibration at the critical pressure $P_{c}$. The maximum of the transverse pressure of a peak is well below the critical pressure $P_{c}$. Therefore a peak is only a local maximum.

For practical purposes the criterium turns out to be quite stringent as inhomogeneous states seen only in the local energy density could be classified as plateaux, but have a transverse pressure maximum well below the transition pressure. Obviously the maximum of the transverse pressure of an inhomogeneous state depends on its initial dotation of local energy density. Moreover there is a fluid distinction between a peak and a plateau as with an addition of local energy density one turns a peak into a plateau or the inverse with depletion. For the criterium the numerical tolerance is set to $20 \%{ }^{3}$ Previously, to classify the simulation one had to integrate the local energy density of the state over spacetime and checking both the maximum and the two minima on the side of the state. With this criterium, one only needs to extract a single value. The criterium makes use of the homogeneous spatial direction, which in our case of planar black branes are the two perpendicular directions $x_{\perp}$, to distinguish between the two types of maxima and minima.

Note that in contrast to the transverse pressure $P_{T}$, the longitudinal pressure $P_{L}$ is not a useful thermodynamical quantity for differentiating between the inhomogeneous states as it correlates with the fluid velocity of the formed peak or plateau. The longitudinal $z$ direction is the dynamical direction of the simulations. Therefore $P_{L}$ is very useful to check how well settled the ongoing simulation is. Once there is no ongoing dynamics, the longitudinal pressure equals up to numerical precision the critical pressure at any position $z$ on the boundary of the spacetime $P_{L}\left(z, t>t_{\text {final }}\right)=P_{c}$. At late times the fluid velocity vanishes, as one reaches the static configuration. This means the longitudinal pressure is constant up to numerical precision once the system is settled.

Criterium for valleys: if at a minimum in local energy density the transverse pressure is near or overshoots the critical equilibrium pressure eq. (3.1), the inhomogeneous state is a valley.

The valley, in analogy to an extended U-shaped formation, has its transverse pressure at the critical pressure $P_{c}$. While the gorge, in analogy to a narrow V-shaped minima formation, has a transverse pressure lower than the critical pressure. The gorge corresponds to a local minima in the local energy density above $\mathcal{E}_{\text {low }}$. In all the simulations the gorge state is rarely realized. It happens intermittently before the merger of peaks or domains, but rarely forms in the reshaping stage as the spinodal instability tends to cool down to the lower stable phase forming valley extents.

\footnotetext{
${ }^{3}$ This is the usual tolerance criterium for the hydrodynamization process (i.e. the time when hydrodynamics applies). It can be stretched to $30 \%$ or reduced to $10 \%$ without much interpretational change.
} 

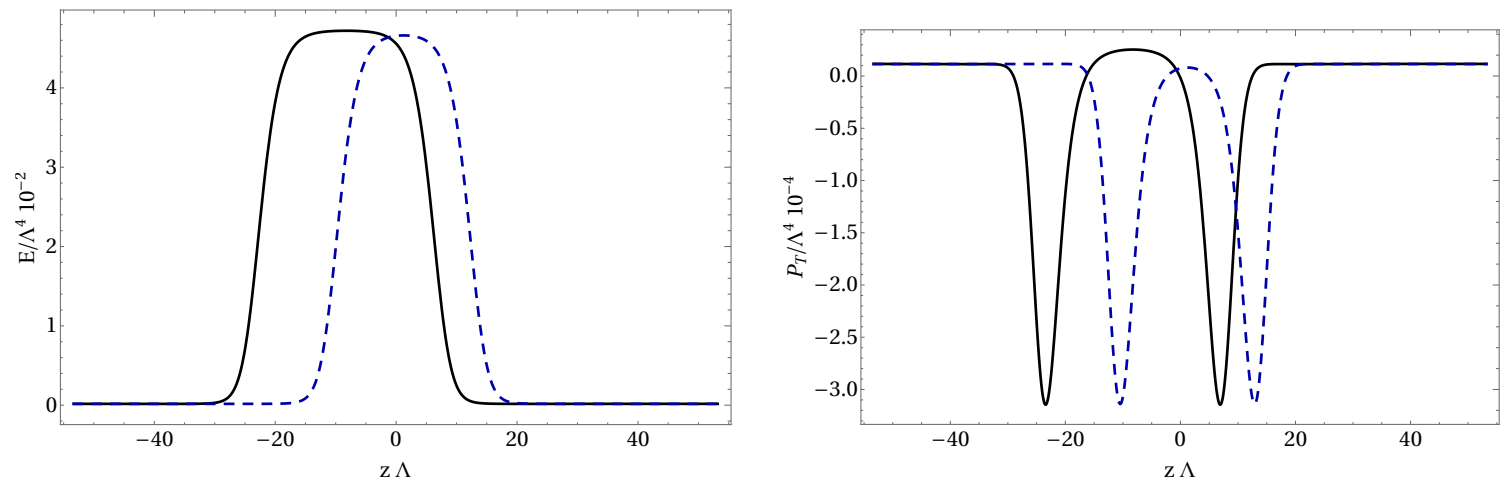

Figure 3. Longitudinal superposed profiles with $\phi_{\mathrm{M}}=2.35$ and $L_{z} \Lambda=107$ for a single plateau in continuous black with initial energy density $\mathcal{E}_{2}$ and for a single peak in dashed blue with initial energy density $\mathcal{E}_{1}$ of (left) the local energy density (right) the transverse pressure.

The presence of a plateaux enforces a phase separated interface, where the transverse pressure inside the plateaux attains the critical pressure $P_{c}$. On the contrary, the maxima of the transverse pressure of a peak does not reach $P_{c}$ and its local energy density might not reach the hot stable phase. The distinction is significant and easy to compute by extracting the corresponding maximum of the transverse pressure. The distinction between peaks and plateaux is of course fluid, since a peak with sufficient local energy density input can be turned into a plateaux. Nevertheless, this new criterium greatly facilitates their distinction and is of interest for the study of the properties of the phase separation especially important as only plateaux have the full phase separation.

\subsection{Final stage}

If the simulated box contains enough total energy density, the final stage of the spinodal instability will be an inhomogeneous phase separated solution (neglecting for now subtleties of quite small finite boxes, where finite size effects act as regulator of the instability [52]) that forms a plateaux. In the special case of not enough total energy density in the system the formed final stage is a single peak.

The transverse pressure of the final stage of a simulation with the initial homogeneous local energy density $\mathcal{E}(t=0)=\mathcal{E}_{2}$ is plotted in the black continuous curve of figure 3 (right). The maximum value of the transverse pressure $P_{T}(z \approx-4.1) \approx 11.0 \times 10^{-5}$ is largely within the tolerance criterium of the critical pressure $\left(\approx 95 \% P_{c}\right)$, therefore it is a plateau. Whereas the maximum of the transverse pressure of the final stage with the same theory but less initial local energy density $\mathcal{E}(t=0)=\mathcal{E}_{1}$ has a value of around $70 \% P_{c}$ below the tolerance criterium, therefore its final inhomogeneous state is a peak. The final longitudinal profile of the transverse pressure is plotted in the blue dashed curve in figure 3 (right). The maximum in $P_{T}$ always corresponds to the location at the longitudinal direction $z$ of the maximum of the local energy density $\mathcal{E}$. As seen in figure 3 (left) the plateau has clearly a large extent near the hot stable phase in the local energy density, while the peak only touches it briefly around its maximum. In both cases (black continuous and blue dashed) the extended minima figure 3 (right) has the value of the critical pressure and 

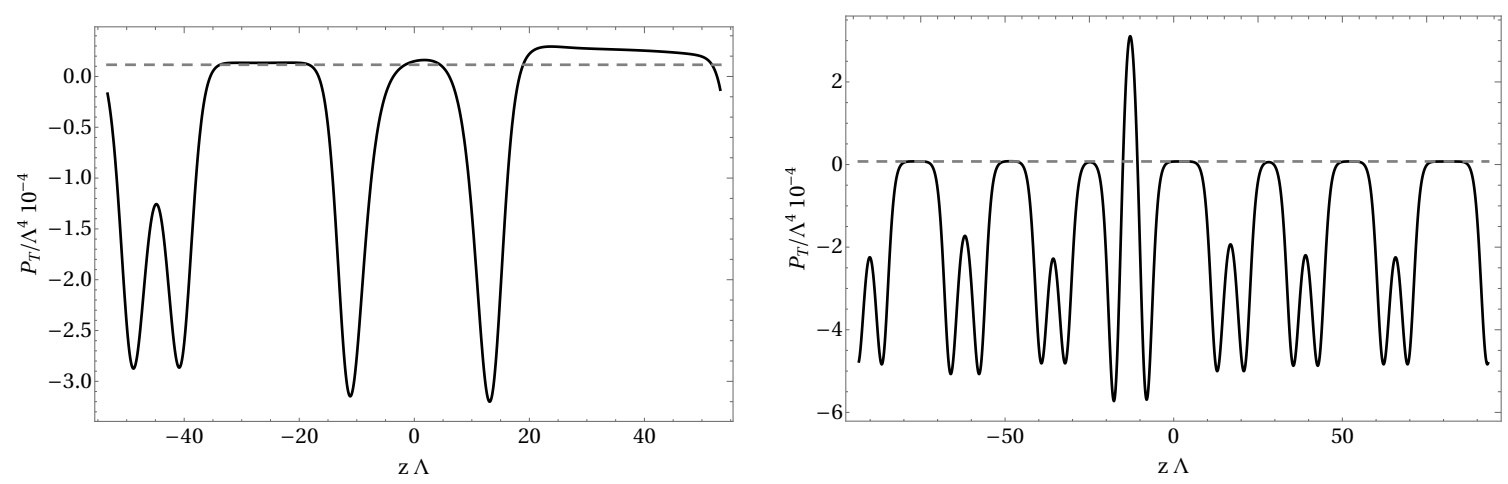

Figure 4. Transverse pressure $P_{L}$ longitudinal profile during reshaping as the solid black line and the critical pressure $P_{c}$ as the dashed gray line (left) with $\phi_{M}=2.35$ at $t \Lambda=1200$ forming a peak, two valleys and a plateau of the simulation visualized on top right of figure 8 (right) with $\phi_{M}=2.3$ at $t \Lambda=1180$ forming several peaks, a single plateau, and valleys in between of the simulation visualized on the left figure 5 .

hence it is a valley. Consequently the simulation in full black is a neat example of a fully phase separated solution.

\subsection{Reshaping stage}

The plateaux are either formed directly early on in the reshaping stage or later on during the merger stage from several joined peaks. Again the longitudinal extent of the simulations is assumed to be wide enough to at least fit a phase separated system with two interfaces. Here are given examples of directly formed peaks and plateaux.

Of course in the early reshaping stage the distinction is by definition a lot messier compared to the settled final stage due to the ongoing dynamics. Nevertheless it is possible to distinguish between peaks and plateaux based on their transverse pressure with the criterium. It explicitly includes the overshooting, compared to the critical pressure, of the maximum of the transverse pressure for a plateau or a valley.

A simple example in the reshaping stage with a single plateau and a single peak is visualized for the local energy density in figure 8 (top right) and for the transverse pressure in figure 4 (left). By extracting the maxima and minima one recognizes in this simulation a single peak at the position $z \Lambda \approx-45$ and a single plateau at $z \Lambda \approx 0$ forming at $t \Lambda=1200$. It also indicates that at this particular moment the valley from $z \Lambda \approx 20$ to $z \Lambda \approx 52$ is also still out-of-equilibrium. The valley between $-35 \lesssim z \lesssim-15$ is much more equilibrated.

In contrast, a much more complicated reshaping simulation can have several maxima. This is demonstrated in an example with a wide extent, where figure 5 (left) shows the evolution of the local energy density and figure 4 (right) the snapshot at the reshaping stage of the transverse pressure. By extracting the maxima and minima one recognizes the formation of seven valleys, a single plateau at the position $z \Lambda \approx-12$ and six peaks, where the transverse pressure maximum is far lower than the transition pressure $P_{c}$. During its reshaping the maximum value of a plateau will severely overshoot the transition pressure $P_{c}$ while the maxima of the peaks do not reach $P_{c}$ during their reshaping. 

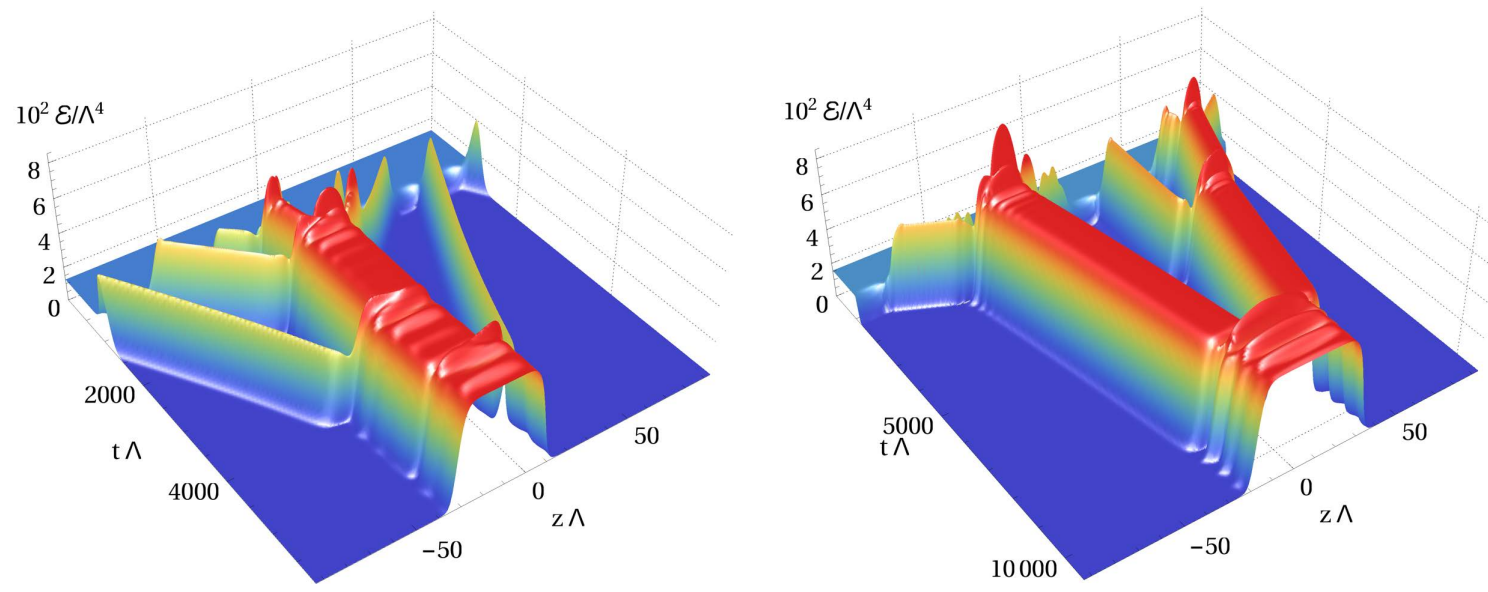

Figure 5. Time evolution of two different merger with $\phi_{\mathrm{M}}=2.3$ and $L_{z} \Lambda \simeq 187$ longitudinal extent: (left) Spacetime evolution $\mathcal{E}(t=0)=\mathcal{E}_{1}$ and with initial $n=1$ perturbation up to $t \Lambda=5885$; (right) Spacetime evolution $\mathcal{E}(t=0)=\mathcal{E}_{2}$ and numerical noise as initial perturbation up to $t \Lambda=10700$.

After reshaping in the merger stage, one observes the peaks to settle quite quickly and have only small changes over time: the maximum energy density of a peak decreases by less than $<1 \%$ over very long times [49]. Relatedly the transverse pressure changes by the same amount over long period times. In the merger stage the formed peaks typically merge together to plateaux. Whereas plateaux take a long time to settle, the more extended the longer and asymptotically tend to $P_{c}$. Therefore the criterium works equally well in out-ofequilibrium during the reshaping and merger stages: by checking the transverse pressure of the inhomogeneous states to be far above or at least attaining a transverse pressure bigger than the tolerance criterium $\left(\geq 20 \% P_{c}\right)$ one is able to handily distinguish plateaux and peaks.

\subsection{Merger stage}

An even more challenging stage for the distinction criterium is the merger stage. In the previous subsections the static final and the early reshaping stage in which each inhomogeneous state form have been discussed. Due to the static nature of the final stage and the usual crisp formation of the spinodal instability those stages are easier to classify. In the sodenominated merger stage the complicated dynamics with the peaks and domains merging happens. Here one discerns that mergers happen in a same qualitative way for the different theories: peak $\leftrightarrow$ peak and peak $\leftrightarrow$ plateau mergers lead to the preferred final solution of a single phase separated plateau. It is worth point out that forming states with more than one plateau requires significant computing resources, due to the large longitudinal extent and the increased evolution time of this large merger and subsequent equilibration. Here I analyse for the first time two evolution, where the inhomogeneous formed states look like out-of-equilibrium or equilibrium plateaux mergers yet are in fact peak $\leftrightarrow$ plateau mergers. The two evolution of the mergers in the figure 5 are a difficult example for the classification of the inhomogeneous states by the criterium. 

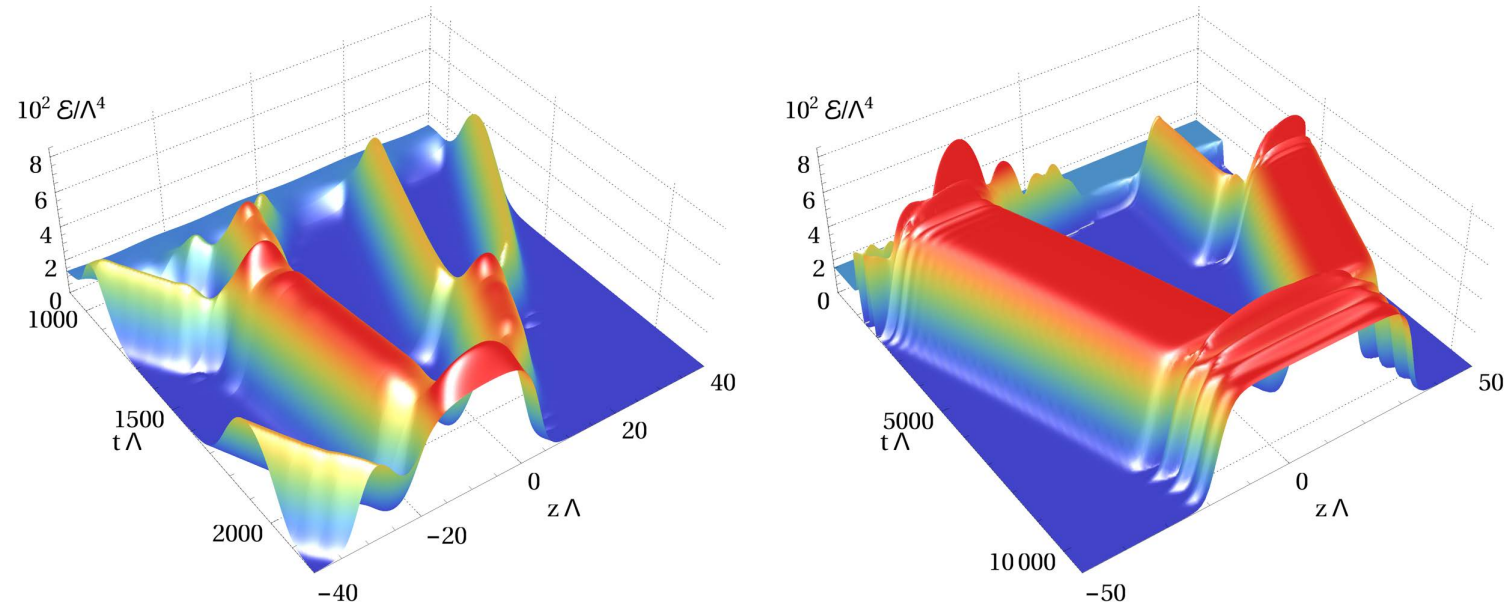

Figure 6. Zoom into the respective spacetime evolution of figure 5 (left) out-of-equilibrium peak $\leftrightarrow$ peak merger (right) Merger of settled peak $\leftrightarrow$ domain.

In the merger of the figure 5 (left) at around $t \Lambda \approx 2000$, one could be tricked into seeing two plateaux merging, while the maximum of the left peak only overshoots early after the left group merges the peaks around $t \Lambda \approx 1400$ the critical pressure, but distinctively settles below. The respective maxima each are a product of mergers and they look deceptively massive enough for being a plateau each. The right merging group of the figure 5 (left) never comes to settling between $1700<t \Lambda<1900$, but as product of only two peaks is very likely a peak too. Hence one can classify in the figure 6 (left) the mergers as being peak $\leftrightarrow$ peak.

In the merger of the figure 5 (right) at around $t \Lambda \approx 8750$, one could be equally tricked into seeing two $\mathcal{E}_{\text {high }}$ plateaux merging. Here the case is a it easier to disentangle as both maxima pick up almost constant velocity towards each other, but settle long before the merger. The left maximum is a plateau, while the right maximum is a peak as its transverse pressure settles at $\approx 0.75 P_{c}$. Consequently the evolution of the figure 6 (right) shows the process of a peak $\leftrightarrow$ plateau merger. Previously demonstrated merger in figure 13 of [49] is happening in out-of-equilibrium where each plateaux is still oscillating around $\mathcal{E}_{\text {high }}$ before merging. Therefore it is a third example of a difficult classification during the merger stage. The left maximum seems to settle quite below the transition pressure, but the right maximum around it. Accordingly it is also a peak $\leftrightarrow$ plateau merger.

Hence the new proposed criterium is useful to classify the inhomogeneous states in all the different stages of the spinodal instability. Only during subsequent fast violent outof-equilibrium mergers the state may not be directly attributable. As conjectured each evolution reaches the same preferred final state, where the extent of the ultimate plateau depends on the total energy density of the calculation.

\section{Characteristics of the interface}

With the clear distinction between different states formed by the spinodal instability, we will now focus on the characteristics of the phase separation with varying criticality. 

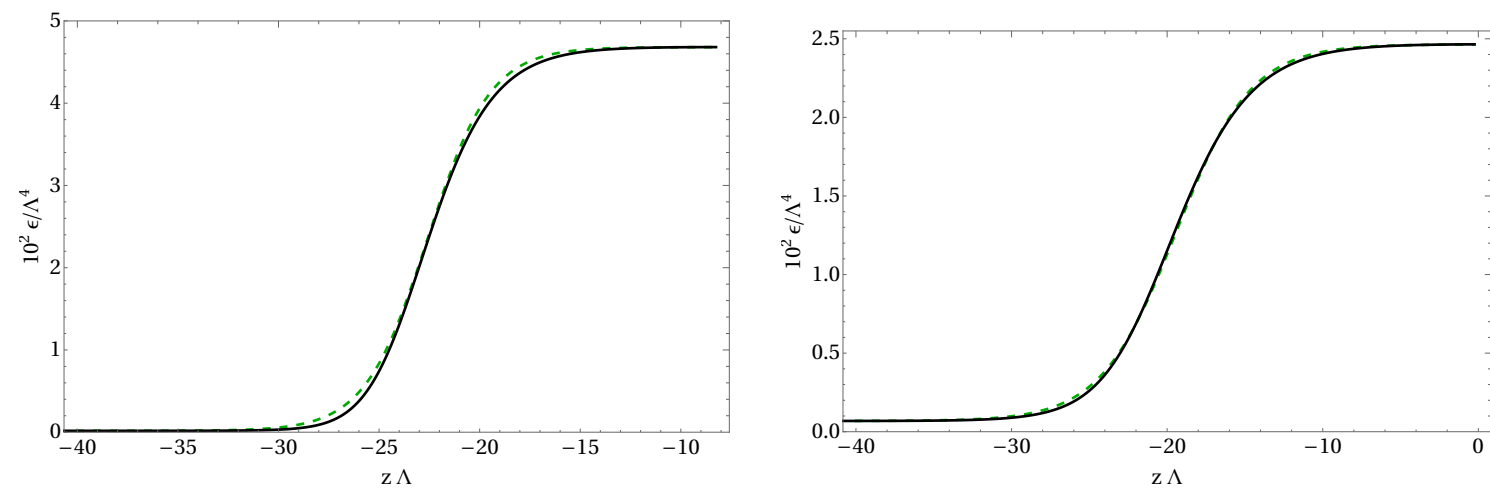

Figure 7. Longitudinal profiles of an interface with (left) $\phi_{\mathrm{M}}=2.35$ and initial energy density $\mathcal{E}_{3}$; (right) $\phi_{\mathrm{M}}=2.45$ and initial energy density $\mathcal{E}_{1}$ both for a single plateau in continuous black with initial energy density $\mathcal{E}_{3}$ and the fitted interface shape in dashed green.

\begin{tabular}{|l|l|l|l|l|l|}
\hline$\phi_{\mathrm{M}}$ & 2.25 & 2.3 & 2.35 & 2.4 & 2.45 \\
\hline$b \Lambda$ & 2.47 & 2.75 & 3.12 & 3.62 & 4.84 \\
\hline
\end{tabular}

Table 2. Approximate width of the plateau interface.

\subsection{Shape}

As seen in figure 7 the shape of the phase transition is well approximated by the function

$$
\mathcal{E}(z) \approx \frac{\Delta \mathcal{E}}{2}\left[1+\tanh \left(\frac{z-z_{0}}{b}\right)\right]+\mathcal{E}_{\text {low }}
$$

with $\Delta \mathcal{E}=\mathcal{E}_{\text {high }}-\mathcal{E}_{\text {low }}, z_{0}$ the exact midpoint between $\mathcal{E}_{\text {low }}$ and $\mathcal{E}_{\text {high }}$ of the interface and $b$ corresponds to the extent of the interface. Note that the fit with eq. (4.1) is very good for the interface from the midpoint $z_{0}$ to $\mathcal{E}_{\text {high }}$, but is only approximate on the downside approach from $z_{0}$ to $\mathcal{E}_{\text {low. }}$. The lower tail fit improves considerably for softer interfaces. This improvement is visible in comparing figure 7 left and right plots. For the interface of the domain wall with a confining vacuum [58] the tanh function is an almost perfect fit [59].

As intuitively given by the picture of a less pronounced first-order phase transition the interface grows with more criticality. There is almost a double increase of the extent going from $\phi_{\mathrm{M}}=2.25$ to $\phi_{\mathrm{M}}=2.45$ as listed in table 2. Counterintuitively, this means the spacetime of the simulated box with the same amount of total energy density needs to be bigger for the subcritical phase transition to fit nicely inside. Nevertheless, it is hot stable energy density $\mathcal{E}_{\text {high }}$ in the studied theories, which is the dominating thermodynamical quantity for determining how much energy a phase separated final plateau has. In all cases roughly more than $90 \%$ of the total energy density concentrate on the domain of the plateaux. While less than $10 \%$ of the total energy density are found in the tails from the midpoint on. Of course, this relates to the small cold energy density $\mathcal{E}_{\text {low }}$ and the comparison assumes also the same finite longitudinal extent. The steepness of the phase transition is the dominating factor leading to only a small portion of the total energy density on the tails of the interface. 


\begin{tabular}{|r|r|r|r|r|r|}
\hline$\phi_{\mathrm{M}}$ & 2.25 & 2.3 & 2.35 & 2.4 & 2.45 \\
\hline $\boldsymbol{\sigma} / \boldsymbol{\Lambda}^{\mathbf{3}}$ & $3.9 \times 10^{-3}$ & $2.9 \times 10^{-3}$ & $1.9 \times 10^{-3}$ & $1.1 \times 10^{-3}$ & $5.4 \times 10^{-4}$ \\
\hline$\left|\mathbf{M i n}\left(\boldsymbol{P}_{\boldsymbol{T}}\right)\right| / \boldsymbol{\Lambda}^{\mathbf{4}}$ & $7.9 \times 10^{-4}$ & $5.3 \times 10^{-4}$ & $3.1 \times 10^{-4}$ & $1.5 \times 10^{-4}$ & $3.4 \times 10^{-5}$ \\
\hline
\end{tabular}

Table 3. Surface tension in equilibrium and the absolute value of the minima of the transverse pressure for each interface.

\subsection{Surface tension}

With increasing criticality, the pressure exercised by the interface decreases. This can be seen both by the decrease of the transverse pressure minima at phase transition and directly by the surface tension of the interface.

The surface tension of the interface is per definition the excess free energy per unit area in the transverse directions $x_{\perp}$. The surface tension of the interface is positive. For an equilibrated homogeneous system, the free energy density per unit volume is equal to the transverse pressure $F(z)=-P_{T}(z)$. As discussed in [49] by integrating the transverse pressure minus the transition pressure over the full extent and taking into account that the final solution has two interfaces, one gets the surface tension of the respective interface

$$
\sigma=\frac{1}{2} \int_{0}^{L_{z}} d z\left[F(z)-F_{c}\right]=-\frac{1}{2} \int_{0}^{L_{z}}\left[P_{T}(z)-P_{c}\right] .
$$

As expected, any presence of an interface increases the free energy of the system.

As listed in table 3, each theory gives rise to a distinct value. The surface tension decreases by almost an order of magnitude from the strongest to the softest first order phase transition. Likewise the minima of the transverse pressure at each interface, as seen in table 3, differ by more than an order of magnitude. This indicates that the range of simulated theories vary widely in criticality.

\section{Evolution of the spinodal instability}

After the outline of the static properties of the inhomogeneous state with varying criticality, I will demonstrate in what follows novel dynamical evolution of the spinodal instability.

\subsection{Comparison of the formation time}

The more critical, meaning the closer the theory is near a critical point, the slower the spinodal instability is. This seems apparent from the different thermodynamics as the unstable/spinodal region shrinks and the magnitude of the negative speed of sound squared decreases too. Summing it up: the more critical the theory is, the range of unstable momenta narrows and the unstable modes have a slower growth rate. The challenge here lies in unveiling this hypothesis in the full non-linear situation of the spinodal dynamics and to show the differences with softer and stronger phase transitions.

As a new measure of the dynamics of the spinodal instability I consider the formation time, defined as the time from the start of the evolution to when the system first reaches 


\begin{tabular}{|l|l|l|l|l|}
\hline$\phi_{\mathrm{M}}$ & 2.25 & 2.35 & 2.4 & 2.45 \\
\hline $\boldsymbol{t}_{\text {formation }} \boldsymbol{\Lambda}$ & 880 & 1380 & 1725 & 2660 \\
\hline
\end{tabular}

Table 4. Formation time of the spinodal instability with varying criticality.

one of the two stable phases. At formation time the inhomogeneous system reaches either $\mathcal{E}_{\text {high }}$ or $\mathcal{E}_{\text {low }}$. As discussed in subsection 3.3, this happens quite far from equilibrium during the reshaping stage. When the system has a large longitudinal extent this can be more complicated, as the stable phase is only reached after the merger of several peaks (seeing each of them is likely to be formed below $\mathcal{E}_{\text {high }}$ ). Moreover, the formation time would depend on the initial conditions and specifically on the initial velocity of the merging peaks. them slower or faster merger. The extraction of the formation time is approximate related to the fact, that one can setup scenarios specifically triggering the most unstable mode (instead of the first mode $n=1$, which populates all unstable modes) or initializing with stronger initial perturbation, where the extracted formation time could be faster.

To extract the formation time and for the sake of comparing different theories, one chooses a subset of simulations, where the spinodal instability directly forms at least one plateau and has similar initial homogeneous local energy density. This setups allow for a direct comparisons of the formation time of the spinodal instability with varying criticality. Due to the simulations having the initial energy density below, but of the similar magnitude of $\mathcal{E}_{\text {high }}$, usually the formation of a plateau with $\mathcal{E}_{\text {high }}$ happens long before the cool down of a valley to $\mathcal{E}_{\text {low }}$. The time where the system reaches both $\mathcal{E}_{\text {low }}$ and $\mathcal{E}_{\text {high }}$ is the full phase separation time. This full separation time depends heavily on the initial state and thus needs a much higher number of different simulations for a meaningful comparison. As both the cooled and the heated regions overshoot the respective equilibrium values of $\mathcal{E}_{\text {low }}$ and $\mathcal{E}_{\text {high }}$ no tolerance criterium is applied for the computation of the formation time. In what follows I extract the direct formation time of a plateau.

The runs visualized in figure 8 provide from all the available simulations the direct plateau formation for each theory with similar initial state. The formation time of the spinodal instability in the simulated theories triples near criticality as documented in the table 4 . In all the simulations of the table 4 the spinodal instability is triggered by the same small initial perturbation. Each simulation has also enough total energy density to form a plateau. Of course the theory with the strongest phase transition with $\phi_{\mathrm{M}}=2.25$ has the fastest formation time. The softest theory near criticality with $\phi_{\mathrm{M}}=2.45$ has a triple longer formation time.

For the theory with $\phi_{\mathrm{M}}=2.3$, no clean direct plateau formation has been simulated, hence it is not listed in the formation table 4. Nevertheless its fastest extracted formation time of $t_{\text {formation }} \Lambda=1190$ fits in the overall trend of the table. In the plateau merger of figure 5 one extracts the fastest respective formation time of $t_{\text {formation }} \Lambda=\{1460,1420\}$ for $\phi_{\mathrm{M}}=2.3$. Due to forming multiple peaks and having to wait for first mergers these formation times are slower and not representative for the corresponding theory. In figure 9 (left) with $\phi_{\mathrm{M}}=2.4$, one gets roughly a formation time of $t_{\text {formation }} \Lambda=2400$ and in figure 9 

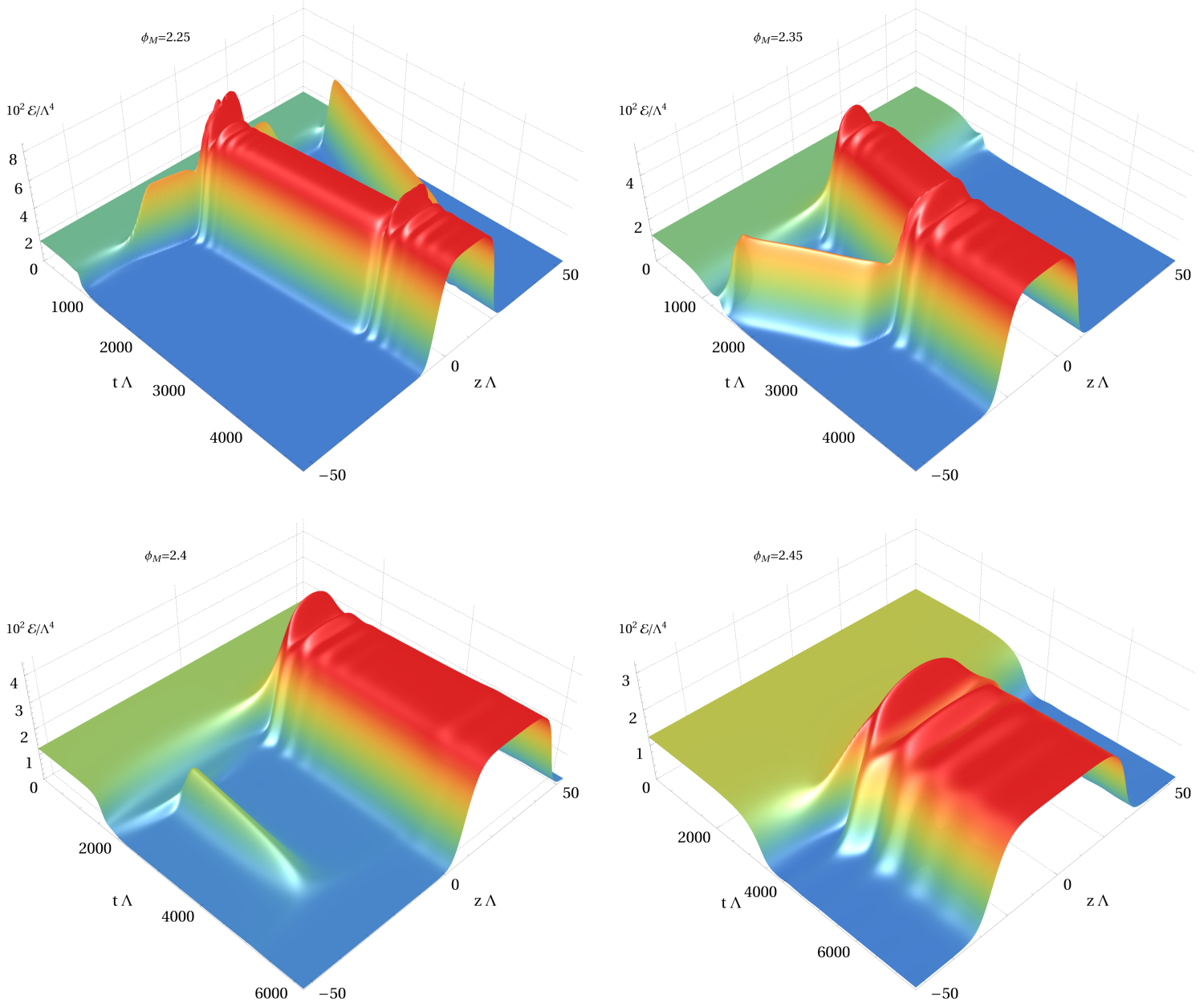

Figure 8. Four spacetime evolution of the local energy density with a plateau formation for the subcritical theories from top left to bottom right with $\phi_{\mathrm{M}}=\{2.25,2.35,2.4,2.45\}$, initial local energy density $\mathcal{E}(t=0)=\left\{\mathcal{E}_{3}, \mathcal{E}_{2}, \mathcal{E}_{2}, \mathcal{E}_{2}\right\}$ and same longitudinal extent $L_{z} \Lambda \approx 107$, where the plateau forms before merger with peaks (top left and right) or the plateau forms with a peak dissipation (bottom left) or only directly a plateau reshapes (bottom right).

(right) with $\phi_{\mathrm{M}}=2.35$ also of $t_{\text {formation }} \Lambda=2400$. Here in both cases the slowdown, relative to the extracted values in table 4 happen due to a double formation of a plateau and a peak. In conclusion for a meaningful comparison of the formation time, one needs to simulate direct single plateau formations. Aptly the strongest phase transition with the largest spinodal region shows the fastest formation time.

\subsection{Peak dissipation into plateaux}

An open puzzle of the holographic simulations of the spinodal instability is the rigidity of the interface. While peaks and plateaux happen to move in the longitudinal extent, their interfaces keep a fixed shape. Even in collisions, the interface of the phase separation only wobbles in a very rigid way. Moreover, only over an extended spacetime have the peaks a slowly varying velocity with almost no distortion of their shape. Here are presented the 

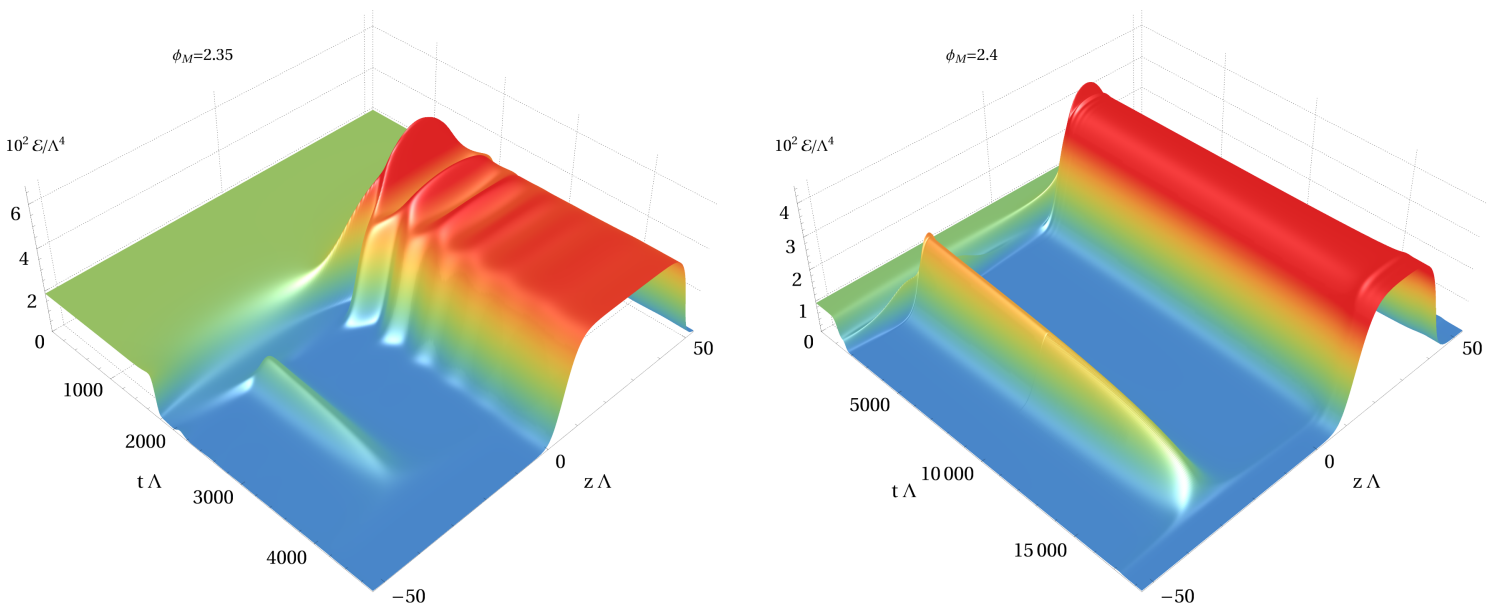

Figure 9. Two subcritical spacetime evolution of the local energy density demonstrating a dissipation of a peak into a plateau with $L_{z} \lambda=107$, (left) initial homogeneous $\mathcal{E}(t=0)=\mathcal{E}_{4}$ and $\phi_{\mathrm{M}}=2.35$ up to $t \Lambda=6250$; (right) initial homogeneous $\mathcal{E}(t=0)=\mathcal{E}_{1}$ and $\phi_{\mathrm{M}}=2.4$ up to $t \Lambda=18350$.

first calculations of a peak dissipating into a plateau for two different theories. This shows the possibility of inhomogeneous states to change their shape.

This process has been newly observed and needs a finely tuned setup. The system needs to directly produce a plateau and a peak both with no initial velocities. In figure 8 (bottom left) and in figure 9 (left + right) are shown three systems for different theories $\phi_{\mathrm{M}}=\{2.35,2.4\}$ and initial energies, but each with enough total energy density to each form a plateau and a peak. A similar fine-tuned setup can also be reproduced in a theory with a very strong first order phase transition with $\phi_{\mathrm{M}}=2.3$. Therefore this untypical dissipation process does not depend on criticality. In the number of simulations [57, 60] performed up to date this process has not yet been observed in large extents, where several peaks or plateaux initially form. While peaks with a slow velocity form on a regular basis it is quite untypical for the spinodal instability to morph into quasi-static features. This happens only if an excited dominant unstable mode carries over from the first stage into the reshaping stage forming a certain number of quasi-static peaks. Then after some little perturbation these peaks usually gain velocity to merge. In this tuned setup, the peak diminishes while staying at the same location.

In figure 8 (bottom left) the simulation first forms both a plateau at around $t \Lambda \approx 1725$ and a peak around $t \Lambda \approx 2250$. Surprisingly, the peak stays at the same spacetime with zero velocity and its maximum energy density decreases first quasi-linear over $t \Lambda \approx 2250$ time duration and then exponentially for $t \Lambda \approx 600$ until it completely disappears. During the quasi-linear stage of the dissipation of the peak, the plateau grows in total energy density, while the valley between the peak and plateau continues to exist. The valley fills up during the exponential stage of the dissipation precipitating the decay of the peak. Afterwards the minimum again cools down to the cold stable phase. After $t \Lambda \approx 5100$ the last amount of local energy density joins and slightly perturbes the plateau. 
In figure 9 (left) one sees first the formation of a plateau at around $t \Lambda \approx 1900$ and a stationary peak around $t \Lambda \approx 2200$. At first the dissipation happens in quasi-linear form during $t \Lambda \approx 1300$ and then exponentially over $t \Lambda \approx 700$. The first stage is quasi-linear as on top of the dissipation proceeds the fast equilibration of the peak after its formation. This dissipation process takes less time as the dissipation in figure 8 (bottom left), as this peak initially has approximately a quarter less total energy density and the quasi-linear dissipation is faster. Again the end of full dissipation of the peak around $t \Lambda \approx 4200$ one notices a slight perturbation of the plateau as the last energy density joins. Here this perturbation is more difficult to discern in the visualization as the plateau itself is still equilibrating from its formation.

In figure 9 (right) one notices the formation of a plateau at around $t \Lambda \approx 1500$ and then of a stationary peak at around $t \Lambda \approx 2050$. While this would be the fastest formation time for $\phi_{\mathrm{M}}=2.4$, the available dataset has no comparable simulations for any other theories with similar initial state, hence it was not considered in table 4 . The third case has the slowest dissipation with the peak only loosing one third of its height over the duration of more than $t \Lambda \approx 10000$. The exponential dissipative stage sets in at around $t \Lambda \approx 12500$. In this simulation the dissipation finishes at $t \Lambda \approx 17500$ and the maximum of the final perturbation of the plateau happens just before at $t \Lambda \approx 17300$.

All three presented atypical cases have a plateau forming before a stationary peak, which dissipates first in linear and than exponentially manner. In conclusion this atypical setup happens for a system with enough total energy density to form a plateau and a peak, but not another inhomogeneous structure. It is striking that the dissipation happens independently of criticality both for harder and softer phase transitions. It is the only process known that defies the rigidity of the interface.

\section{Discussion}

Simulating the spinodal instability far from and near a critical point, one recognizes that the harder the first order phase transition is the more dramatic the spinodal instability is. The shrinking extent and the harder surface tension of the interface of the phase separation go pair in pair. New insights from the dynamical simulations are the rising formation time, while showcasing similar merger dynamics with varying degree of criticality.

Looking at the holographic dynamics of the spinodal instability one remarks: the more pronounced the first-order phase transition, the wider the unstable momenta range is which dictates a faster spinodal instability. This translates in a much faster formation time away from criticality. With decreasing criticality the domain wall between the stable phases shrinks. As the magnitudes between the local energy densities $\mathcal{E}_{\text {low }}$ and $\mathcal{E}_{\text {high }}$ grows with less criticality, one needs more local energy density in the system to generate a phase separated solution. The finding of this single preferred final solution [49] is now substantiated with softer and harder first-order phase transitions. The newly proposed criterium for the distinction of this inhomogeneous state preforms well in all stages of the spinodal instability. It fittingly distinguishes between the phase separated plateaux and prevalent local maxima, so named peaks. 
Moreover the newly found atypical setup with a peak dissipating into a plateaux is a progress in the understanding of the rigidity of the interface. For the first time, an inhomogeneous solution has been shown to change its shape after formation without directly merging. The peak without velocity slowly dissipates. This setup shows that the interface is not fully immovable. It is still surprising how rigid the interface is across the theories with widely different criticality. An open question remains to simulate a collision with high velocities, where the remnants would maybe separate again post collision. For now, all scenarios of the spinodal instability demonstrate peaks joining together with no side remnants. A possible scenario involves a peak with high speed crossing a plateau. It would also be interesting to be able to trigger the spinodal instability in a plasma blob created by shockwave collisions near a critical point. The numerical challenge here lies in the extreme slow down of the dynamics at a critical point [51]. A different future line of work involves computing the quasinormal mode spectra of the gravity solutions $[12,15,61-63]$ of each theory as their linearized responses allow insights on the phase structure and the timescales like the formation times.

Although this approach to criticality used a bottom-up model, the performed simulations suggest that the qualitative physics may be quite universal in strong coupling situations approaching a critical point where the negative speed of sound induces the spinodal instability. It would be interesting to break the planar symmetry to allow dynamics in the transversal directions. While the reshaping stage dynamics may change, the simulated formation times will be similar to this study. Extending the simulations to include conserved charges such as the chemical baryonic potential $\mu$ would be interesting to enhance the analysis to the full $T \mu$ phase diagram $[28,32,35]$ and make contact with the experimental programs: a major goal for heavy-ion collisions is to determine the existence of a first-order phase transition between hadronic and quark-gluon plasma in the QCD phase diagram [64, 65], which is predicted by numerous effective field theory models [66]. As the first order transition presumably has a big temperature range it is worthwhile to explore if the heavy-ion experiments see a signature [67] of it. Very recently a sophisticated hadronic mean-field simulation using the Vlasov equation has also shown that hadronic systems initialized in unstable regions of the phase diagram undergo spontaneous spinodal decomposition [68]. It will be exciting to see the upcoming comparisons with experimental data influenced by the two phase system.

Previously we have demonstrated that the spinodal instability is very well described by a second-order hydrodynamics [49] with second-order purely spatial gradients included and redefined non-conformal second-order transport coefficients. It would be very interesting to develop the new hydrodynamics formulation of [69, 70], which may be able to incorporate the large spatial gradients of the phase separation for a full hydrodynamical evolution.

\section{Acknowledgments}

I am grateful for fruitful discussions with J. Casalderrey-Solana, E. Kiritsis and D. Mateos; Y. Bea, J. Brewer, N. Jokela, A. Vuorinen and M. Zilhao for interesting exchanges and R. Janik, M. Jarvinen, M. Stephanov and L. Yin for general discussions on the critical 
point and phase transitions. I thank the Center for Supercomputing of Galicia (CESGA) for providing extensive High Performance Computing resources in the Finisterra cluster (project usciemat). I would like to thank the Institute for Nuclear Theory for their hospitality at the INT-19-1b workshop during early stages of this work. I acknowledge support through H2020-MSCA-IF-2019 ExHolo 898223. This work is also supported by the "María de Maeztu" Units of Excellence program MDM-2016-0692, Xunta de Galicia (Consellería de Educación) and the Spanish Research State Agency.

Open Access. This article is distributed under the terms of the Creative Commons Attribution License (CC-BY 4.0), which permits any use, distribution and reproduction in any medium, provided the original author(s) and source are credited.

\section{References}

[1] R.A. Janik and R.B. Peschanski, Asymptotic perfect fluid dynamics as a consequence of AdS/CFT, Phys. Rev. D 73 (2006) 045013 [hep-th/0512162] [INSPIRE].

[2] J.L. Albacete, Y.V. Kovchegov and A. Taliotis, Modeling Heavy Ion Collisions in AdS/CFT, JHEP 07 (2008) 100 [arXiv:0805.2927] [INSPIRE].

[3] D. Grumiller and P. Romatschke, On the collision of two shock waves in AdS $S_{5}$, JHEP 08 (2008) 027 [arXiv: 0803.3226] [INSPIRE].

[4] S.S. Gubser, S.S. Pufu and A. Yarom, Entropy production in collisions of gravitational shock waves and of heavy ions, Phys. Rev. D 78 (2008) 066014 [arXiv:0805.1551] [InSPIRE].

[5] P.M. Chesler and L.G. Yaffe, Holography and colliding gravitational shock waves in asymptotically AdS spacetime, Phys. Rev. Lett. 106 (2011) 021601 [arXiv:1011.3562] [INSPIRE].

[6] J. Casalderrey-Solana, M.P. Heller, D. Mateos and W. van der Schee, From full stopping to transparency in a holographic model of heavy ion collisions, Phys. Rev. Lett. 111 (2013) 181601 [arXiv: 1305.4919] [INSPIRE].

[7] J. Casalderrey-Solana, M.P. Heller, D. Mateos and W. van der Schee, Longitudinal Coherence in a Holographic Model of Asymmetric Collisions, Phys. Rev. Lett. 112 (2014) 221602 [arXiv: 1312.2956] [INSPIRE].

[8] P.M. Chesler and L.G. Yaffe, Holography and off-center collisions of localized shock waves, JHEP 10 (2015) 070 [arXiv:1501.04644] [INSPIRE].

[9] P.M. Chesler, Colliding shock waves and hydrodynamics in small systems, Phys. Rev. Lett. 115 (2015) 241602 [arXiv: 1506.02209] [INSPIRE].

[10] R.A. Janik, G. Plewa, H. Soltanpanahi and M. Spalinski, Linearized nonequilibrium dynamics in nonconformal plasma, Phys. Rev. D 91 (2015) 126013 [arXiv:1503.07149] [INSPIRE].

[11] A. Buchel and A. Day, Universal relaxation in quark-gluon plasma at strong coupling, Phys. Rev. D 92 (2015) 026009 [arXiv: 1505.05012] [INSPIRE].

[12] A. Buchel, M.P. Heller and R.C. Myers, Equilibration rates in a strongly coupled nonconformal quark-gluon plasma, Phys. Rev. Lett. 114 (2015) 251601 [arXiv:1503.07114] [INSPIRE]. 
[13] R. Rougemont, A. Ficnar, S. Finazzo and J. Noronha, Energy loss, equilibration, and thermodynamics of a baryon rich strongly coupled quark-gluon plasma, JHEP 04 (2016) 102 [arXiv: 1507.06556] [INSPIRE].

[14] M. Attems et al., Thermodynamics, transport and relaxation in non-conformal theories, JHEP 10 (2016) 155 [arXiv:1603.01254] [INSPIRE].

[15] M. Attems et al., Holographic Collisions in Non-conformal Theories, JHEP 01 (2017) 026 [arXiv: 1604.06439] [INSPIRE].

[16] A. Czajka et al., Bulk Viscosity at Extreme Limits: From Kinetic Theory to Strings, JHEP 07 (2019) 145 [arXiv: 1807.04713] [INSPIRE].

[17] A. Czajka et al., On bulk viscosity at weak and strong 't Hooft couplings, Mod. Phys. Lett. A 35 (2020) 2030012 [arXiv: 1807.07950] [INSPIRE].

[18] M. Attems et al., Paths to equilibrium in non-conformal collisions, JHEP 06 (2017) 154 [arXiv: 1703.09681] [INSPIRE].

[19] A. Bzdak, S. Esumi, V. Koch, J. Liao, M. Stephanov and N. Xu, Mapping the Phases of Quantum Chromodynamics with Beam Energy Scan, Phys. Rept. 853 (2020) 1 [arXiv: 1906. 00936] [INSPIRE].

[20] V. Friese, The CBM experiment at GSI/FAIR, Nucl. Phys. A 774 (2006) 377 [InSPIRE].

[21] M. Durante et al., All the fun of the FAIR: fundamental physics at the facility for antiproton and ion research, Phys. Scripta 94 (2019) 033001 [arXiv: 1903.05693] [INSPIRE].

[22] M.A. Stephanov, K. Rajagopal and E.V. Shuryak, Signatures of the tricritical point in QCD, Phys. Rev. Lett. 81 (1998) 4816 [hep-ph/9806219] [INSPIRE].

[23] M.A. Stephanov, K. Rajagopal and E.V. Shuryak, Event-by-event fluctuations in heavy ion collisions and the QCD critical point, Phys. Rev. D 60 (1999) 114028 [hep-ph/9903292] [INSPIRE].

[24] J. Erlich, E. Katz, D.T. Son and M.A. Stephanov, QCD and a holographic model of hadrons, Phys. Rev. Lett. 95 (2005) 261602 [hep-ph/0501128] [INSPIRE].

[25] O. DeWolfe, S.S. Gubser and C. Rosen, A holographic critical point, Phys. Rev. D 83 (2011) 086005 [arXiv: 1012.1864] [INSPIRE].

[26] C. Athanasiou, K. Rajagopal and M. Stephanov, Using Higher Moments of Fluctuations and their Ratios in the Search for the QCD Critical Point, Phys. Rev. D 82 (2010) 074008 [arXiv: 1006.4636] [INSPIRE].

[27] P. Alba et al., Effect of the QCD equation of state and strange hadronic resonances on multiparticle correlations in heavy ion collisions, Phys. Rev. C 98 (2018) 034909 [arXiv: 1711.05207] [INSPIRE].

[28] R. Critelli, J. Noronha, J. Noronha-Hostler, I. Portillo, C. Ratti and R. Rougemont, Critical point in the phase diagram of primordial quark-gluon matter from black hole physics, Phys. Rev. D 96 (2017) 096026 [arXiv: 1706.00455] [INSPIRE].

[29] J. Brewer, S. Mukherjee, K. Rajagopal and Y. Yin, Searching for the QCD critical point via the rapidity dependence of cumulants, Phys. Rev. C 98 (2018) 061901 [arXiv:1804.10215] [INSPIRE]. 
[30] R. Rougemont, R. Critelli and J. Noronha, Nonhydrodynamic quasinormal modes and equilibration of a baryon dense holographic QGP with a critical point, Phys. Rev. D 98 (2018) 034028 [arXiv: 1804.00189] [INSPIRE].

[31] R. Critelli, R. Rougemont and J. Noronha, Holographic Bjorken flow of a hot and dense fluid in the vicinity of a critical point, Phys. Rev. D 99 (2019) 066004 [arXiv:1805.00882] [INSPIRE].

[32] L. Du, U. Heinz, K. Rajagopal and Y. Yin, Fluctuation dynamics near the QCD critical point, Phys. Rev. C 102 (2020) 054911 [arXiv: 2004.02719] [InSPIRE].

[33] M.-W. Li, Y. Yang and P.-H. Yuan, Analytic Study on Chiral Phase Transition in Holographic QCD, JHEP 02 (2021) 055 [arXiv:2009.05694] [INSPIRE].

[34] C. Hoyos, N. Jokela, M. Jarvinen, J.G. Subils, J. Tarrio and A. Vuorinen, Transport in strongly coupled quark matter, Phys. Rev. Lett. 125 (2020) 241601 [arXiv:2005.14205] [INSPIRE].

[35] T. Dore, J. Noronha-Hostler and E. McLaughlin, Far-from-equilibrium search for the QCD critical point, Phys. Rev. D 102 (2020) 074017 [arXiv:2007.15083] [INSPIRE].

[36] D. Mroczek, A.R. Nava Acuna, J. Noronha-Hostler, P. Parotto, C. Ratti and M.A. Stephanov, Quartic cumulant of baryon number in the presence of a QCD critical point, Phys. Rev. C 103 (2021) 034901 [arXiv:2008.04022] [InSPIRE].

[37] M. Nahrgang and M. Bluhm, Modeling the diffusive dynamics of critical fluctuations near the QCD critical point, Phys. Rev. D 102 (2020) 094017 [arXiv:2007.10371] [InSPIRE].

[38] V. Dexheimer, J. Noronha, J. Noronha-Hostler, C. Ratti and N. Yunes, Future physics perspectives on the equation of state from heavy ion collisions to neutron stars, J. Phys. G 48 (2021) 073001 [arXiv: 2010.08834] [INSPIRE].

[39] J.M. Maldacena, The Large $N$ limit of superconformal field theories and supergravity, Adv. Theor. Math. Phys. 2 (1998) 231 [Int. J. Theor. Phys. 38 (1999) 1113] [hep-th/9711200] [INSPIRE].

[40] A. Buchel, A Holographic perspective on Gubser-Mitra conjecture, Nucl. Phys. B 731 (2005) 109 [hep-th/0507275] [INSPIRE].

[41] R. Gregory and R. Laflamme, Black strings and p-branes are unstable, Phys. Rev. Lett. 70 (1993) 2837 [hep-th/9301052] [INSPIRE].

[42] R. Emparan and H.S. Reall, A Rotating black ring solution in five-dimensions, Phys. Rev. Lett. 88 (2002) 101101 [hep-th/0110260] [INSPIRE].

[43] R. Emparan and H.S. Reall, Black Rings, Class. Quant. Grav. 23 (2006) R169 [hep-th/0608012] [INSPIRE].

[44] R. Emparan, T. Harmark, V. Niarchos and N.A. Obers, World-Volume Effective Theory for Higher-Dimensional Black Holes, Phys. Rev. Lett. 102 (2009) 191301 [arXiv:0902.0427] [INSPIRE].

[45] R. Emparan, T. Harmark, V. Niarchos and N.A. Obers, Essentials of Blackfold Dynamics, JHEP 03 (2010) 063 [arXiv:0910.1601] [INSPIRE].

[46] P. Figueras, M. Kunesch and S. Tunyasuvunakool, End Point of Black Ring Instabilities and the Weak Cosmic Censorship Conjecture, Phys. Rev. Lett. 116 (2016) 071102 [arXiv: 1512.04532] [INSPIRE]. 
[47] M. Attems, Y. Bea, J. Casalderrey-Solana, D. Mateos, M. Triana and M. Zilhao, Phase Transitions, Inhomogeneous Horizons and Second-Order Hydrodynamics, JHEP 06 (2017) 129 [arXiv: 1703.02948] [INSPIRE].

[48] R.A. Janik, J. Jankowski and H. Soltanpanahi, Real-Time dynamics and phase separation in a holographic first order phase transition, Phys. Rev. Lett. 119 (2017) 261601 [arXiv: 1704.05387] [INSPIRE].

[49] M. Attems, Y. Bea, J. Casalderrey-Solana, D. Mateos and M. Zilhão, Dynamics of Phase Separation from Holography, JHEP 01 (2020) 106 [arXiv: 1905.12544] [INSPIRE].

[50] L. Bellantuono, R.A. Janik, J. Jankowski and H. Soltanpanahi, Dynamics near a first order phase transition, JHEP 10 (2019) 146 [arXiv:1906.00061] [INSPIRE].

[51] M. Attems, Y. Bea, J. Casalderrey-Solana, D. Mateos, M. Triana and M. Zilhão, Holographic Collisions across a Phase Transition, Phys. Rev. Lett. 121 (2018) 261601 [arXiv: 1807.05175] [INSPIRE].

[52] Y. Bea et al., Crossing a large- $N$ phase transition at finite volume, JHEP 02 (2021) 061 [arXiv: 2007.06467] [INSPIRE].

[53] H. Bantilan, P. Figueras and D. Mateos, Real-time Dynamics of Plasma Balls from Holography, Phys. Rev. Lett. 124 (2020) 191601 [arXiv:2001.05476] [InSPIRE].

[54] M. Bianchi, D.Z. Freedman and K. Skenderis, Holographic renormalization, Nucl. Phys. B 631 (2002) 159 [hep-th/0112119] [inSPIRE].

[55] P.M. Chesler and L.G. Yaffe, Numerical solution of gravitational dynamics in asymptotically anti-de Sitter spacetimes, JHEP 07 (2014) 086 [arXiv: 1309.1439] [INSPIRE].

[56] W. van der Schee, Gravitational collisions and the quark-gluon plasma, Ph.D. thesis, Utrecht U., 2014. arXiv:1407.1849 [INSPIRE].

[57] M. Attems, Holographic approach of the spinodal instability to criticality, Zenodo dataset, v20210308 (Mar, 2021) DOI.

[58] O. Aharony, S. Minwalla and T. Wiseman, Plasma-balls in large $N$ gauge theories and localized black holes, Class. Quant. Grav. 23 (2006) 2171 [hep-th/0507219] [InSPIRE].

[59] M. Jarvinen, private communication.

[60] M. Attems, Y. Bea, J. Casalderrey-Solana, D. Mateos and M. Zilhao, Dynamics of phase separation from holography, Zenodo dataset, v20190918 (Sept, 2019) DOI.

[61] P.K. Kovtun and A.O. Starinets, Quasinormal modes and holography, Phys. Rev. D 72 (2005) 086009 [hep-th/0506184] [INSPIRE].

[62] R.A. Janik, J. Jankowski and H. Soltanpanahi, Quasinormal modes and the phase structure of strongly coupled matter, JHEP 06 (2016) 047 [arXiv: 1603.05950] [INSPIRE].

[63] T. Alho, J. Remes, K. Tuominen and A. Vuorinen, Quasinormal modes and thermalization in improved holographic QCD, Phys. Rev. D 101 (2020) 106025 [arXiv:2002.09544] [InSPIRE].

[64] K. Fukushima and T. Hatsuda, The phase diagram of dense QCD, Rept. Prog. Phys. 74 (2011) 014001 [arXiv: 1005.4814] [INSPIRE].

[65] K. Fukushima and C. Sasaki, The phase diagram of nuclear and quark matter at high baryon density, Prog. Part. Nucl. Phys. 72 (2013) 99 [arXiv:1301.6377] [inSPIRE]. 
[66] M.A. Stephanov, QCD phase diagram and the critical point, Prog. Theor. Phys. Suppl. 153 (2004) 139 [hep-ph/0402115] [INSPIRE].

[67] M. Bluhm et al., Dynamics of critical fluctuations: Theory - phenomenology - heavy-ion collisions, Nucl. Phys. A 1003 (2020) 122016 [arXiv: 2001.08831] [INSPIRE].

[68] A. Sorensen and V. Koch, Phase transitions and critical behavior in hadronic transport with a relativistic density functional equation of state, arXiv:2011.06635 [INSPIRE].

[69] F.S. Bemfica, M.M. Disconzi and J. Noronha, Causality and existence of solutions of relativistic viscous fluid dynamics with gravity, Phys. Rev. D 98 (2018) 104064 [arXiv: 1708.06255] [INSPIRE].

[70] P. Kovtun, First-order relativistic hydrodynamics is stable, JHEP 10 (2019) 034 [arXiv: 1907.08191] [INSPIRE]. 